\title{
Trajectories of Change in an Open-access Internet-Based Cognitive Behavior Program for Childhood and Adolescent Anxiety: Open Trial
}

Sonja March ${ }^{1}$, BPsych(Hons), BA(Hons), PhD; Philip J Batterham², BSc(Hons), MPH, PhD; Arlen Rowe, BPsych(Hons), PhD; Caroline Donovan ${ }^{4}$, BA(Hons), PhD; Alison L Calear ${ }^{2}$, BAppPsych(Hons), PhD; Susan H Spence $^{4,5}$, BSc(Hons), MBA, PhD

${ }^{1}$ Centre for Health Research and School of Psychology and Counselling, University of Southern Queensland, Springfield, Australia

${ }^{2}$ Centre for Mental Health Research, Research School of Population Health, The Australian National University, Canberra, Australia

${ }^{3}$ Centre for Health Research, University of Southern Queensland, Springfield, Australia

${ }^{4}$ School of Applied Psychology, Griffith University, Mt Gravatt, Australia

${ }^{5}$ Australian Institute for Suicide Prevention, Griffith University, Mt Gravatt, Australia

\section{Corresponding Author:}

Sonja March, BPsych(Hons), BA(Hons), PhD

Centre for Health Research and School of Psychology and Counselling

University of Southern Queensland

Sinnathamby Blvd

Springfield, 4300

Australia

Phone: 61734704434

Email: sonja.march@usq.edu.au

\section{Abstract}

Background: Although evidence bolstering the efficacy of internet-based cognitive behavioral therapy (iCBT) for treating childhood anxiety has been growing continuously, there is scant empirical research investigating the timing of benefits made in iCBT programs (eg, early or delayed).

Objective: This study aims to examine the patterns of symptom trajectories (changes in anxiety) across an iCBT program for anxiety (BRAVE Self-Help).

Methods: This study's participants included 10,366 Australian youth aged 7 to 17 years (4140 children aged 7-12 years; 6226 adolescents aged 12-17 years) with elevated anxiety who registered for the BRAVE Self-Help program. Participants self-reported their anxiety symptoms at baseline or session 1 and then at the commencement of each subsequent session.

Results: The results show that young people completing the BRAVE Self-Help program tend to fall into two trajectory classes that can be reliably identified in terms of high versus moderate baseline levels of anxiety and subsequent reduction in symptoms. Both high and moderate anxiety severity trajectory classes showed significant reductions in anxiety, with the greatest level of change being achieved within the first six sessions for both classes. However, those in the moderate anxiety severity class tended to show reductions in anxiety symptoms to levels below the elevated range, whereas those in the high symptom group tended to remain in the elevated range despite improvements.

Conclusions: These findings suggest that those in the high severity group who do not respond well to iCBT on a self-help basis may benefit from the additional support provided alongside the program or a stepped-care approach where progress is monitored and support can be provided as necessary.

(JMIR Ment Health 2021;8(6):e27981) doi: 10.2196/27981

\section{KEYWORDS}

iCBT; child; adolescent; anxiety; online; trajectories of change 


\section{Introduction}

Anxiety is an increasingly common childhood mental health condition, with potentially significant life-long repercussions for those who do not access early treatment [1]. Internet-based or eHealth interventions are recommended for encouraging the primary prevention and early intervention for mental illness [2]. In particular, the evidence base for internet-based cognitive behavior therapy (iCBT) in the treatment of childhood anxiety has been growing continuously, with the best outcomes observed when delivered with therapist support [3-8]. More recently, research has demonstrated the feasibility of self-help iCBT for childhood anxiety as a means of achieving widespread service implementation in real-world clinical and community contexts [9]. Self-help iCBT, which does not rely on any therapist support, has the potential to offer evidence-based mental health assistance in a timely and effective manner and in a way that overcomes barriers to accessibility, cost, stigma, anonymity, and shortages of health care professionals [10]. Several successful iCBT platforms have been developed and validated internationally for children and young people (eg, BIP Anxiety [11,12] and SPARX [13]) and adults (eg, MindSpot Clinic [14], This Way Up [15], and Shuti [16,17]).

The BRAVE Self-Help program is the only widely disseminated iCBT program for childhood anxiety, which is made publicly available, free of charge, to all children and adolescents in Australia. Although the preliminary effects of this self-help iCBT program appear somewhat weaker than those found for the therapist-supported iCBT version [9], clinically meaningful improvements are still made by a large proportion of anxious youth who engage in and complete the program, further demonstrating its potential as a population-level, early intervention. Specifically, it was demonstrated that for those users who completed six or more self-help iCBT sessions, there was a moderate to large reduction in anxiety (Cohen $d=0.81$ and Cohen $d=0.87$ for adolescents and children, respectively [9]). Furthermore, for those who had completed nine sessions, approximately $57.7 \%$ (94/163) achieved recovery into nonelevated levels of anxiety and 54.6\% (89/163) showed statistically reliable reductions in anxiety [9]. However, anxiety was not measured at every session, and the amount of change made from session to session was not explicitly tested. Therefore, it is unclear when reductions in anxiety actually occurred and for whom.

Very little research has been conducted on the timing of benefits made in iCBT programs (eg, early or delayed). March et al [9] demonstrated that users' anxiety was reduced after only three self-help iCBT sessions (Cohen $d=0.59$ ), suggesting that change may occur early and rapidly. Furthermore, in their examination of brief and full versions of a cognitive behavioral therapy (CBT) website (MoodGym) for adults with elevated depression, Christensen et al [18] found that a single module of CBT was insufficient to reduce depression symptoms, but extended CBT (approximately three sessions) was associated with greater improvements. Importantly, however, they also found that programs longer than three sessions were not necessarily associated with greater improvement [18]. There is also some evidence from face-to-face interventions that can provide potential insight into the ideal dosage and response trajectories. In one study of face-to-face CBT for anxious youth, a nonlinear symptom trajectory was reported, whereby a rapid response was evident over the first six sessions, with tapering anxiety reductions over the remainder of the intervention [19]. In another study examining the separate trajectories of anxiety and depressive symptoms over the course of a transdiagnostic treatment for adolescents, Queen et al [20] found that, on average, participants' total anxiety scores reduced steadily during treatment (by 4.76 units every 8 weeks) and then slowed during the follow-up period (reduced by only 1.48 units every 8 weeks). Together, the evidence to date suggests that a reduction in symptoms appears to commence after at least three sessions of iCBT [9,18]. However, a session-by-session trajectory across iCBT programs for children and adolescents has yet to be examined.

We examined patterns of symptom trajectories (changes in anxiety) across BRAVE Self-Help program sessions to determine whether BRAVE Self-Help produces a gradual and linear impact on anxiety symptoms or whether the greatest impacts are made early in treatment and to determine distinct trajectories of anxiety symptoms within BRAVE Self-Help participants. Such information will help in determining ideal doses of self-help iCBT and whether small doses of treatment are sufficient. Given that variability is likely to be present in how young people respond to self-help interventions, identifying factors associated with different trajectories may assist in understanding the mechanisms through which the program works and may inform targeting and tailoring of the program. Thus, we aim to identify subgroups of participants (including identifying common demographic and clinical characteristics of these subgroups) based on latent change trajectories in anxiety scores in response to BRAVE Self-Help. Previous studies have used this analytic approach to determine the trajectories of depressive symptoms and suicidal ideation in internet-based interventions [17,21], and in longitudinal studies examining trajectories of anxiety symptoms [22,23]; however, this approach is yet to be applied for examining responses to anxiety treatment in the context of a web-based program for young people. The identification of key demographic and clinical variables that predict subgroup membership has the potential to inform the effective delivery of supported interventions, tailored, or alternative treatments. In this way, the results will inform recommendations regarding the use of iCBT within the context of population-level models of care that are open to everyone and are typically not monitored by health care professionals.

\section{Methods}

\section{Intervention}

BRAVE Self-Help is an interactive internet-based iCBT program for preventing and treating anxiety among youth. The program, described extensively elsewhere [9,24], is offered as an open-access web-based program targeting young Australians aged 7 to 17 years. It comprises 10 sessions of 30 to 60 minutes each, with two additional booster sessions that can be completed as revision modules. Sessions include CBT techniques incorporated into interactive web-based activities focused on 
psychoeducation, recognition of physiological symptoms of anxiety, relaxation training, cognitive strategies of coping statements and cognitive restructuring, graded exposure, problem-solving approaches, self-reinforcement, and relapse prevention. Each session comprised pages that included the presentation of information or material, examples of technique application, activities to facilitate the knowledge acquisition and application of skills to the young person's circumstances, quizzes to consolidate learning, and homework activities to promote the application of skills in real-world contexts. The self-help program has previously demonstrated its acceptability and feasibility [9].

Depending on age, participants completed either the child program (7-12 years) or the adolescent program (12-17 years), with 12-year-olds given a choice of either program. Although there were accompanying parent programs, these were not included in this study. BRAVE Self-Help is delivered without any therapist support, and there are no timing restrictions between sessions; however, the sessions must be completed in a predetermined sequence. Automatic reminders to complete sessions are sent to a young person via email.

\section{Participants}

Participants included 10,366 Australian youth aged 7 to 17 years with elevated anxiety (4140 children aged 7-12 years; 6226 adolescents aged 12-17 years) who registered for the BRAVE Self-Help program between July 1, 2014, and October 26, 2018. Table 1 presents a full summary of the baseline characteristics of participants according to program grouping.

Table 1. BRAVE Self-Help baseline participant characteristics ( $\mathrm{N}=10,366)$.

\begin{tabular}{|c|c|c|c|}
\hline Characteristics & Child program $(n=4140)$ & Adolescent program $(n=6226)$ & Total participants $(\mathrm{N}=10,366)$ \\
\hline Age (years), mean (SD) & $9.34(1.48)$ & $14.55(1.66)$ & $12.47(3.01)$ \\
\hline \multicolumn{4}{|l|}{ Gender, n (\%) } \\
\hline Male & $2192(52.95)$ & $1480(23.77)$ & $3672(35.42)$ \\
\hline Female & $1948(47.05)$ & $4532(72.79)$ & $6480(62.52)$ \\
\hline Other & $0(0)$ & $214(3.44)$ & $214(2.06)$ \\
\hline Transgender or transsexual & $0(0)$ & $40(0.64)$ & $40(0.39)$ \\
\hline Transgender or transsexual & $0(0)$ & $12(0.19)$ & $12(0.12)$ \\
\hline Genderqueer & $0(0)$ & $59(0.95)$ & $59(0.57)$ \\
\hline Androgynous & $0(0)$ & $28(0.45)$ & $28(0.27)$ \\
\hline None of the above & $0(0)$ & $75(1.21)$ & $75(0.71)$ \\
\hline \multicolumn{4}{|l|}{ Remoteness area, n (\%) } \\
\hline Major cities & $2324(56.14)$ & $3559(57.16)$ & $5883(56.75)$ \\
\hline Inner regional & $1013(24.47)$ & $1424(22.87)$ & $2437(23.51)$ \\
\hline Outer regional & 499 (12.05) & $712(11.44)$ & $1211(11.68)$ \\
\hline Remote & $111(2.68)$ & $141(2.27)$ & $252(2.43)$ \\
\hline Very remote & $38(0.92)$ & $20(0.32)$ & $58(0.56)$ \\
\hline Missing & $155(3.74)$ & $370(5.94)$ & $525(5.07)$ \\
\hline Number of sessions completed, mean (SD) & $3.04(2.98)$ & $2.04(2.51)$ & $2.39(2.67)$ \\
\hline \multicolumn{4}{|l|}{ Baseline anxiety } \\
\hline $\mathrm{CAS}-8^{\mathrm{a}}$, mean $(\mathrm{SD})$ & $14.16(3.04)$ & $15.75(3.42)$ & $15.11(3.36)$ \\
\hline Elevated, n (\%) & $2275(54.95)$ & $2819(45.28)$ & $5094(49.14)$ \\
\hline Clinical, n (\%) & $1865(45.05)$ & $3407(54.72)$ & $5272(50.86)$ \\
\hline
\end{tabular}

${ }^{a}$ CAS-8: Children's Anxiety Scale 8-item.

The BRAVE Self-Help program is an open-access intervention offered to young people and families throughout Australia; thus, registration does not require referral from a health care professional. Children and adolescents who registered for the program were referred by school-based professionals (3480/10,366, 33.57\%); referred by external health professionals (1973/10,366, 19.03\%); referred by parents, friends, or family members $(1330 / 10,366,12.83 \%)$; referred through beyondblue $(645 / 10,366,6.22 \%)$; self-referred through internet searching
$(840 / 10,366,8.1 \%)$; or referred through other means (eg, word-of-mouth, radio, magazine, or advertising; 676/10,366, $6.52 \%$ ). A full breakdown of referral sources is provided in Multimedia Appendix 1.

Eligible participants were required to have registered for the BRAVE Self-Help program between July 2014 and October 2018 and to have completed the Children's Anxiety Scale 8-item (CAS-8) [25] during the registration process. All participants 
had access to BRAVE Self-Help (single-cohort longitudinal design); there was no randomization or comparison condition. Participants were not required to demonstrate symptomatic levels of anxiety to register for the program; however, only those with elevated anxiety were included in this study. Of the participants included in this study, 49.14\% (5094/10,366) showed elevated levels of anxiety (CAS- $8 \geq 84$ th percentile or T-score $\geq 60)$ and $50.89 \%(5272 / 10,366)$ showed clinical levels of anxiety (CAS- $8 \geq 96$ th percentile or T-score $\geq 65$ ). As the primary objective was to examine the trajectories of session completion and reductions in anxiety, there were no inclusion criteria regarding the minimum number of completed program sessions.

\section{Measures}

All measures were embedded within the BRAVE Self-Help program. Further data were collected via BRAVE program analytics regarding adherence to the program.

\section{Sociodemographics}

Basic demographic information, including age, gender, and location (postcode), was collected during the registration process. Age was measured in years, and the residential location was assessed using postcode. Postcode data were coded according to the Australian Standard Geographic Classification system [26], and participants were grouped into the categories of major cities, inner regional, outer regional, remote, and very remote locations. In terms of gender, participants were able to select from male, female, transgender or transsexual, transgender or transsexual, genderqueer, androgynous, or other. For the purposes of analysis, gender was subsequently recoded into male, female, and other.

\section{Anxiety Severity}

Anxiety levels were measured using the CAS-8 [25]. The CAS-8 comprises eight items asking the young person to rate how often each item (eg, "I worry what other people think of me") applied to them. Items are rated on a 4-point scale ranging from 0 (never) to 3 (always). Items are summed to produce a total score ranging from 0 to 24 , with higher scores indicating greater levels of anxiety.

The CAS- 8 has been used in large-scale school-based prevention program trials with subsequent population-level, gender-standardized norms for comparison [25]. Scores $\leq 83 \mathrm{rd}$ percentile ( $\leq \mathrm{T}$-score 59: CAS- 8 score $\leq 9$ for males and 11 for females) are considered normal; scores $\geq 84$ th percentile (above T-score 60: CAS- 8 score $\geq 10$ for males and 12 for females) are considered indicative of elevated anxiety and scores $\geq 94$ th percentile (above T-score 65: CAS- 8 score $\geq 13$ for males and 16 for females) indicate clinical levels of anxiety. For this study, and as per procedures of March et al [9], for other gender, scores $\geq 11$ were considered indicative of elevated and scores $\geq 15$ were considered indicative of clinical anxiety. The CAS-8 was completed by participants at baseline or session 1 and then at the commencement of each subsequent session (ie, up to nine time points after baseline). For example, if a participant had completed five sessions, they would have CAS- 8 data at baseline and sessions 2, 3, 4, and 5. The CAS-8 has demonstrated good psychometric properties, with a reliability coefficient $\alpha$ of .89 in previous studies [25] and an average internal consistency across data collection points of 0.83 in this study.

\section{Program Adherence}

Program adherence was measured as the number of sessions completed by each participant over a period of 20 weeks from the date of registration. The number of sessions completed was automatically recorded by the program.

\section{Procedure}

Data for this study were collected as a part of a large community effectiveness trial of the BRAVE Self-Help program (ethical approval H13REA264 from the governing university). During the registration process, participants were required to read a developmentally appropriate web-based participant information sheet and provide informed consent. Children aged below 16 years were also required to obtain parental consent to continue with registration. After providing consent, participants created a profile and completed an initial questionnaire, including basic demographic data (age, gender, location, referral source, and email contact address) and baseline anxiety assessment (CAS-8). Participation in the program was voluntary, and participants could choose to provide anonymous information (eg, nickname) if they chose. They could also discontinue using the program or withdraw from the study at any time without adverse consequences.

Participant data were recorded for a 20 -week period from the date of registration, thus allowing sufficient time for the completion of the 10 sessions. Figure 1 provides a visual representation of the selection process for the inclusion and exclusion of data and the final sample included in the study. 
Figure 1. Participant inclusion in the BRAVE Self-Help program. CAS-8: Children's Anxiety Scale, 8-item.

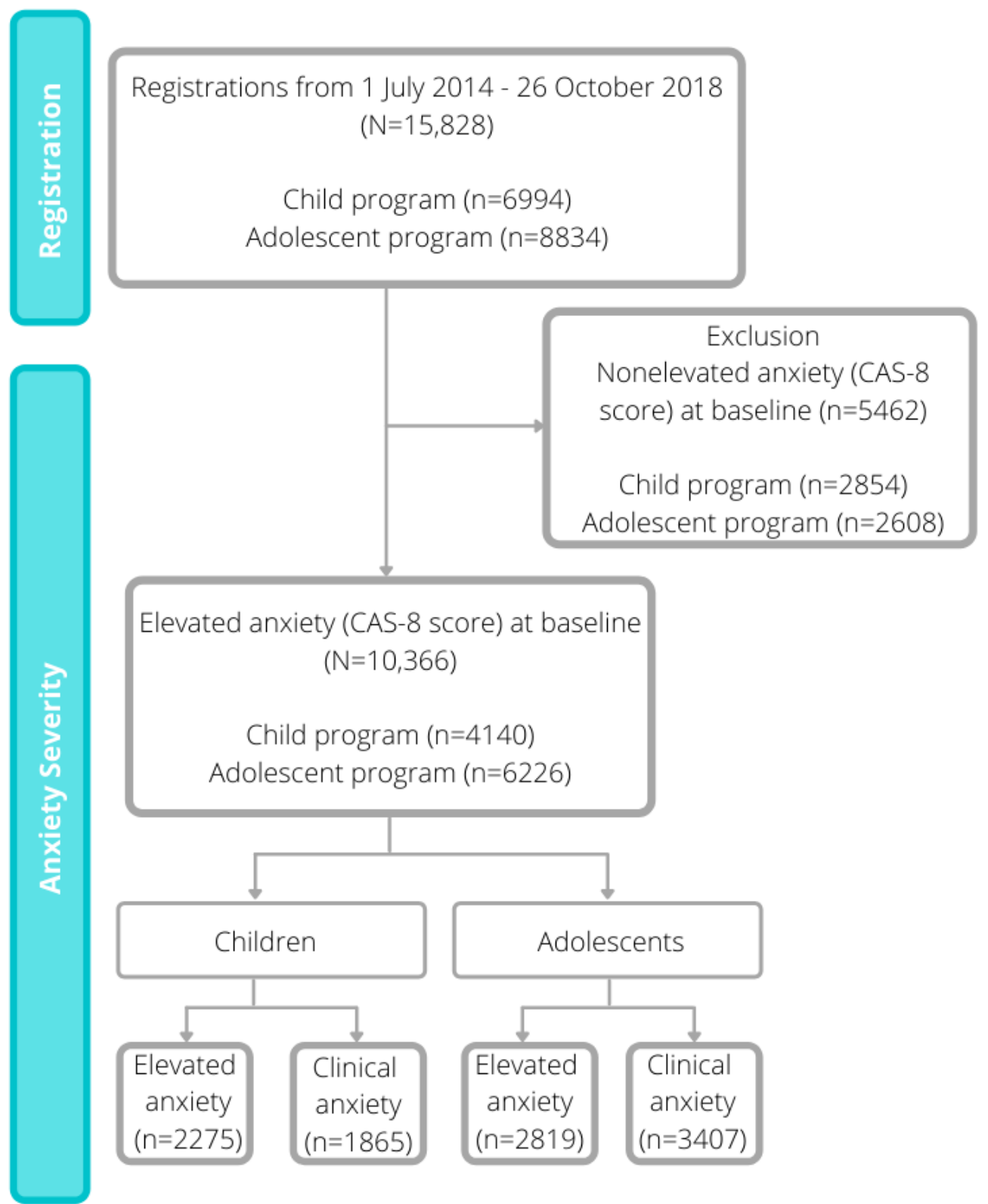

\section{Analytic Strategy}

Trajectories of anxiety symptoms based on CAS-8 scores were identified using growth mixture models (GMMs), with intercept, linear change, and quadratic change estimated using all available data from the baseline assessment and up to nine subsequent measurement occasions. GMM was used to identify latent classes of participants with distinct longitudinal trajectories, with all available data included in the models [27]. The GMM approach is a form of latent class analysis that identifies distinct subgroups, combined with growth modeling that estimates linear and quadratic trajectories over time. The methodology classifies the heterogeneity of individual trajectories in symptoms over time into a discrete number of latent classes. Models with between one and five latent classes were tested to identify an optimal number of latent classes based on a significant Bootstrapped Likelihood Ratio Test (BLRT) [28]. We also considered the size of each latent class, as models with classes that represent $<5 \%$ of the total sample are difficult to identify [28]. After selecting an optimal number of classes, predictors of latent class membership were tested using logistic regression models to identify participants' characteristics (eg, baseline anxiety severity, gender, age, and geographic location), who showed differential responses to the intervention, and to characterize the relationship between adherence and response to the BRAVE Self-Help program. GMM analyses were conducted using Mplus version 7 (Muthén \& Muthén), whereas descriptive and regression analyses were conducted using SPSS version 25 (IBM Corp). 


\section{Results}

\section{Program Adherence}

Figure 2. Rates of program adherence. CAS-8: Children's Anxiety Scale, 8-item.

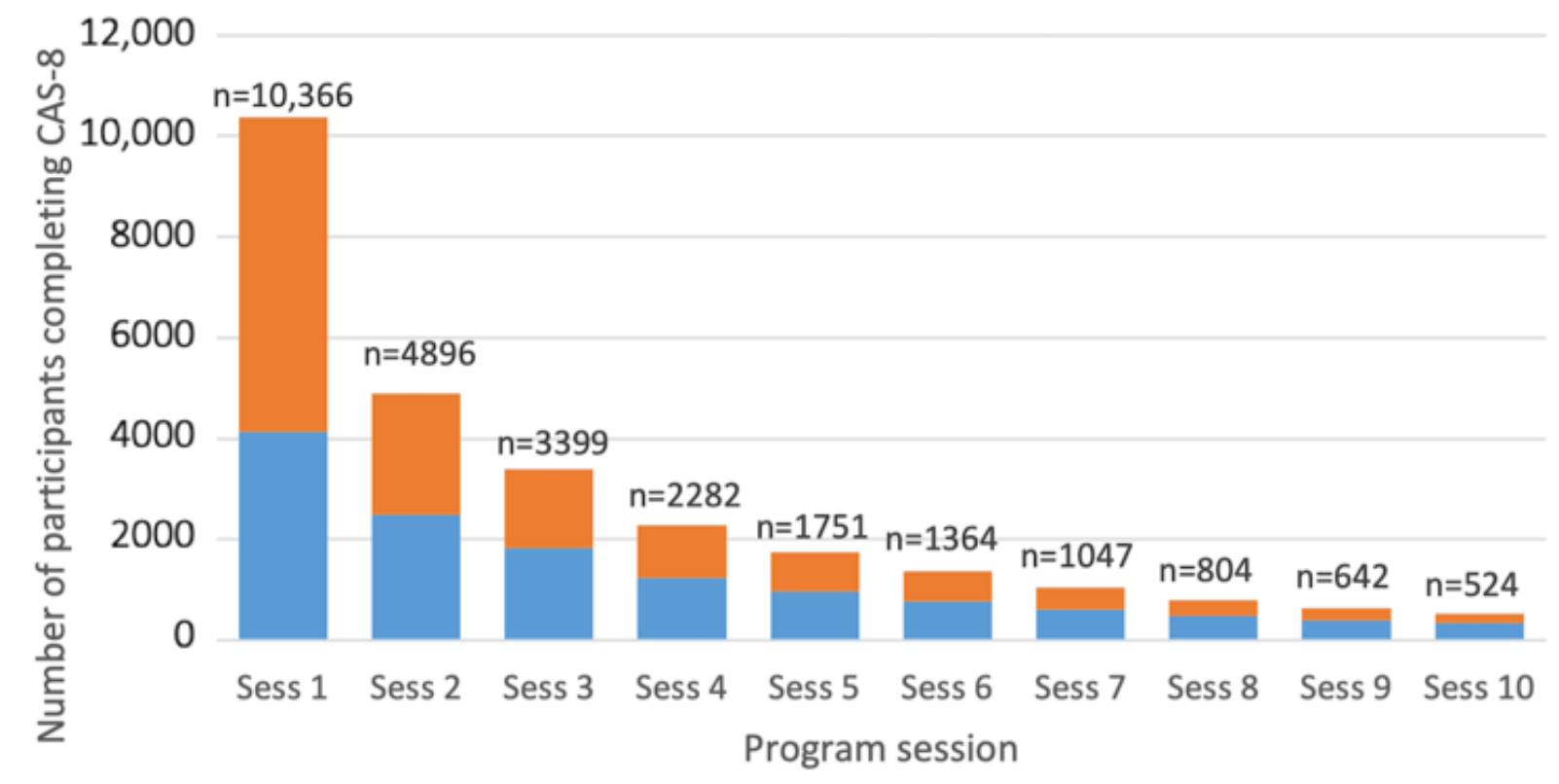

\section{Trajectories of Anxiety Symptoms}

The GMM with two classes had a significantly better fit than a single-class solution based on the BLRT statistic $(P<.001)$; however, the BLRT for a three-class model was not significant as compared with the two-class model $(P=.99)$. Furthermore, in the three-class model, one of the classes accounted for $0.7 \%$ $(73 / 10,366)$ of the sample. Consequently, a two-class model was selected. Scores on the CAS-8 for the two total classes are shown in Figure 3. On the basis of the observed trajectories, the two classes were labeled as high anxiety severity (HAS;
The number of participants completing each session decreased throughout the program. As indicated in Figure 2, only half of the participants completed two sessions of the program.

\section{Child program adolescent program}

$2280 / 10,366,21.99 \%$ of the sample, shown in orange), and moderate anxiety severity (MAS; 8086/10,366, 78.01\% of the sample, shown in blue). On the basis of the GMM model, the intercept for the CAS-8 scores in the HAS group was 19.72 (SE 0.09 ), with a negative slope (estimate $=-1.39$; SE $0.11 ; P<.001$ ). The intercept for the MAS group was 13.75 (SE 0.04), with a negative slope (estimate $=-0.97$; SE $0.04 ; P<.001$ ). A quadratic relationship was also evident in both the HAS (estimate $=0.08$; $P<.001$ ) and MAS (estimate $=0.05 ; P<.001$ ) groups, showing that the rate of change in CAS- 8 scores decreased over time for both classes. 
Figure 3. Trajectories of anxiety symptoms for the two latent classes of high and moderate anxiety severity based on observed mean (SD). CAS-8: Children's Anxiety Scale, 8-item.

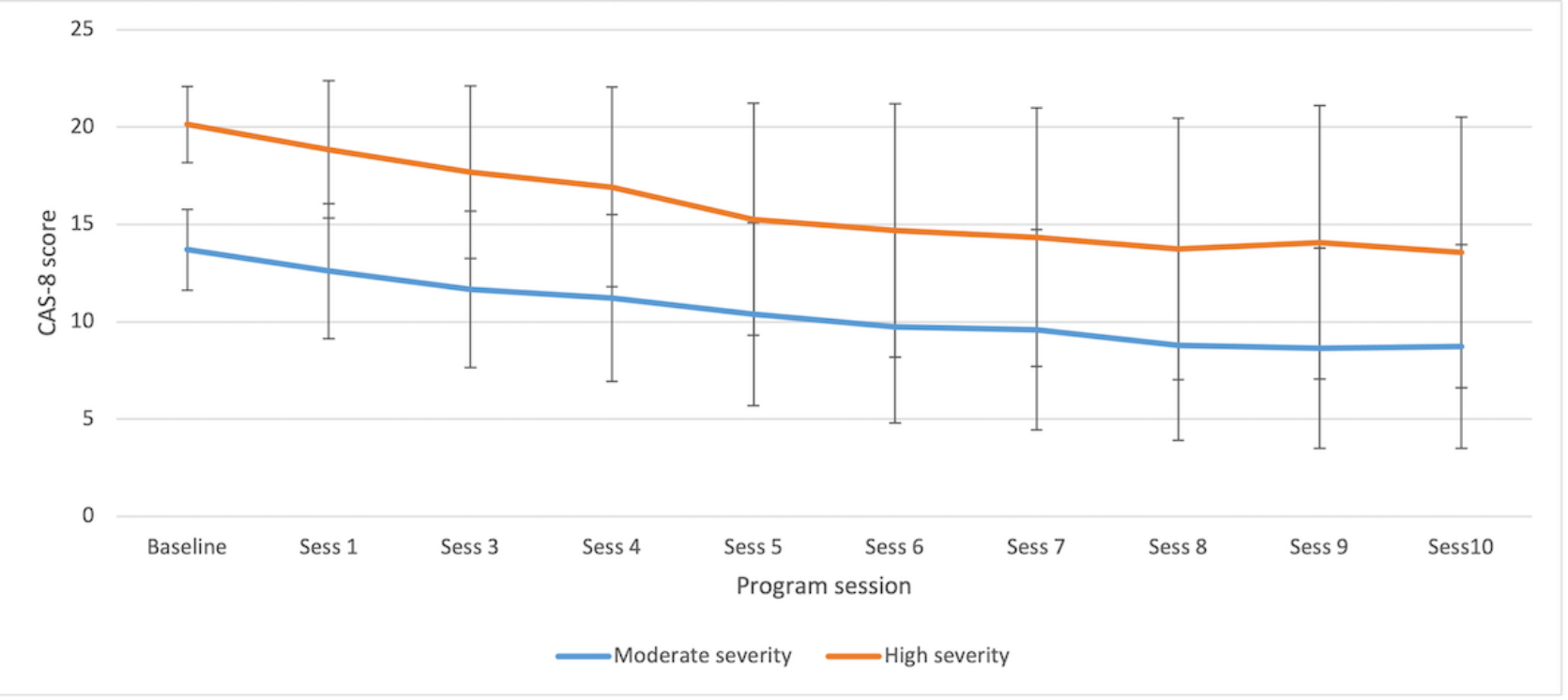

\section{Predictors of Class Membership}

A logistic regression model was used to identify the factors associated with class membership. The outcomes are presented in Table 2. Teen program participants had $31 \%$ greater odds of being in the HAS class than those receiving the child program $(P<.001)$. For every year increase in age, participants were $4 \%$ more likely to fall in the HAS class $(P=.007)$. Females had twice greater odds of being in the HAS class than males
$(P<.001)$. Participants of other gender had $47 \%$ greater odds of being in the HAS class than females $(P=.007)$. The effects of remoteness of residence were not significant (Table 2). In terms of program adherence, those participants who completed a greater number of sessions were less likely to be classified as HAS, with the likelihood of being in the HAS class decreasing by $3 \%$ with the completion of each additional session $(P=.01)$. There were no effects on the date of registration or the number of activities completed in session 1. 
Table 2. Logistic regression model of class membership: odds of having a high compared with moderate anxiety severity class trajectory.

\begin{tabular}{|c|c|c|c|c|}
\hline Variable & Estimate (SE) & Chi-square $(d f)$ & $P$ value & Odds ratio $(95 \% \mathrm{CI})$ \\
\hline Program: teen versus child & $0.27(0.05)$ & $29.9(1)$ & $<.001^{\mathrm{a}}$ & 1.31 (1.19 to 1.44$)$ \\
\hline Age at registration & $0.04(0.02)$ & $7.2(1)$ & .007 & 1.04 (1.01 to 1.07$)$ \\
\hline Gender & & $148.5(2)$ & $<.001$ & \\
\hline Male versus female & $-0.69(0.06)$ & $135.8(1)$ & $<.001$ & $0.50(0.45$ to 0.56$)$ \\
\hline Other versus female & $0.38(0.14)$ & $7.2(1)$ & .007 & $1.47(1.11$ to 1.95$)$ \\
\hline State of residence & & $17.3(8)$ & .03 & \\
\hline Missing versus $\mathrm{SA}^{\mathrm{b}}$ & $-0.44(0.50)$ & $0.8(1)$ & .38 & $0.64(0.24$ to 1.71$)$ \\
\hline $\mathrm{ACT}^{\mathrm{c}}$ versus $\mathrm{SA}$ & $-0.10(0.19)$ & $0.3(1)$ & .57 & $0.90(0.62$ to 1.30$)$ \\
\hline $\mathrm{NSW}^{\mathrm{d}}$ versus $\mathrm{SA}$ & $-0.23(0.09)$ & $6.0(1)$ & .01 & 0.79 (0.66 to 0.95$)$ \\
\hline Northern Territory versus SA & $0.18(0.28)$ & $0.4(1)$ & .52 & $1.19(0.69$ to 2.05$)$ \\
\hline Queensland versus SA & $-0.28(0.10)$ & $8.5(1)$ & .004 & $0.75(0.62$ to 0.91$)$ \\
\hline Tasmania versus SA & $-0.40(0.18)$ & $5.0(1)$ & .02 & $0.67(0.47$ to 0.95$)$ \\
\hline Victoria versus SA & $-0.33(0.10)$ & $11.3(1)$ & .001 & $0.72(0.59$ to 0.87$)$ \\
\hline Western Australia versus SA & $-0.31(0.11)$ & $8.4(1)$ & .004 & $0.73(0.59$ to 0.91$)$ \\
\hline Remoteness area & & $4.0(5)$ & .54 & \\
\hline Missing versus major city & $0.18(0.117)$ & $2.8(1)$ & .10 & $1.20(0.97$ to 1.48$)$ \\
\hline Inner regional versus major city & $-0.01(0.064)$ & $0.0(1)$ & .93 & $0.99(0.88$ to 1.12$)$ \\
\hline Outer regional versus major city & $-0.01(0.084)$ & $0.0(1)$ & .90 & $0.99(0.85$ to 1.16$)$ \\
\hline Remote area versus major city & $-0.05(0.168)$ & $0.1(1)$ & .78 & $0.96(0.69$ to 1.32$)$ \\
\hline Very remote area versus major city & $-0.37(0.398)$ & $0.9(1)$ & .34 & $0.69(0.32$ to 1.49$)$ \\
\hline Date of registration & $-0.01(0.021)$ & $7.4(1)$ & .45 & $0.99(0.95$ to 1.02$)$ \\
\hline Number of sessions completed & $-0.03(0.011)$ & $12.2(1)$ & .01 & $0.97(0.95$ to 0.99$)$ \\
\hline Activities completed in session 1 & $0.00(0.005)$ & $1.5(1)$ & .40 & $1.00(0.99$ to 1.01$)$ \\
\hline Constant & $4.49(8.44)$ & $0.3(1)$ & .59 & $\mathrm{~N} / \mathrm{A}^{\mathrm{e}}$ \\
\hline
\end{tabular}

${ }^{\mathrm{a}}$ Italics indicate statistical significance $(P<.05)$.

${ }^{\mathrm{b}} \mathrm{SA}$ : South Australia.

${ }^{\mathrm{c}}$ ACT: Australian Capital Territory.

dNSW: New South Wales.

e N/A: not applicable.

\section{Discussion}

\section{Principal Findings}

This study examined the trajectories of responses to a web-based, open-access, self-help intervention for youth anxiety. The results suggested two classes of trajectories that could be distinguished by high versus moderate baseline levels of anxiety and levels of change over time. Irrespective of trajectory, participants, on average, obtained benefits from the program, with participants generally showing significant linear reductions in anxiety. From a baseline average of around 20 (>98th percentile for females and males), those in the HAS trajectory class showed reduced anxiety scores of 6.58 points (or 5.68 points based on model estimates that accounted for missing data) on the CAS-8; however, this level remained within the elevated range. In contrast, the MAS trajectory class showed reductions of 3.87 points (or 4.31 points based on model estimates) on the CAS- 8 from a baseline of 12.6 (84th percentile for females; 94th percentile for males) to a level in the nonelevated range. Indeed, on average, those in the MAS class obtained a clinically meaningful benefit (scores within the nonelevated range) by session 6 .

The finding that the HAS class tended to remain in the elevated range of anxiety scores despite showing significant reductions from a very high starting point suggests that at least a proportion of this group may require additional assistance over and above the self-help iCBT program. The finding also revealed that the HAS class completed fewer sessions than their MAS counterparts. It is possible that lower levels of perceived symptom improvement among participants in the HAS class contributed to lower motivation to continue engaging with the program, or a greater difficulty completing sessions due to 
ongoing symptom severity and difficulty implementing strategies. Thus, a therapist-supported model or stepped-care approach may be beneficial for this group, whereby HAS participants either receive additional support from the outset or are monitored (in terms of anxiety reduction and session compliance) and are referred for additional therapist support as necessary. HAS participants could be easily identified at the beginning of the program (due to higher initial scores), and subsequently referred, monitored, and provided with additional support or intervention elements if their response to the program was poor. Preliminary results from case studies of a stepped-care model of BRAVE highlight the potential of this approach [29].

The study results also suggested that the rate of anxiety reduction decreased over time for both trajectory classes, with greater reductions occurring in the first six sessions than in later sessions. Importantly, in line with the findings of Christensen et al [18], the results showed that young people can show significant reductions in symptoms after brief iCBT programs. The findings are also partially consistent with the findings of Chu et al [19] for face-to-face CBT for youth anxiety; however, Chu et al [19] showed a more rapid initial decrease and a clearer plateau than this study. The results are also consistent with those of Queen et al [20], who demonstrated a large reduction in symptoms during the first eight weeks of face-to-face treatment and a far smaller drop in symptoms in the second eight weeks of follow-up treatment. It would seem that around six sessions of iCBT may be sufficient for many users, with the option of further session completion for those who choose to do so, or for whom poor treatment response suggests they may be indicated. Importantly, with the knowledge that six sessions may be sufficient for many users, this provides an opportunity to develop shorter intervention programs that may be more appealing and motivating for young people and ultimately attract more users.

In terms of predictors of class membership, the study found that adolescents and females were more likely to be in the HAS class compared with MAS class than children and males. These findings are perhaps unsurprising given that anxiety levels are known to increase with age and are higher among women [30]. Given that the HAS class tended to complete fewer sessions and were less likely to reach a nonelevated status, it is important that the greater representation by females and adolescents is considered to ensure that the needs of these participants are being considered in program design and delivery. For example, our previous research indicated that adolescents are more likely to complete the program alone without parental support. It is possible that additional forms of support, whether from a web-based therapist, school teacher, peer coach, or even automated chatbot could enhance treatment outcomes for adolescents. It may also be feasible to build web-based reward systems designed for adolescents that provide enhanced support to increase motivation and program adherence. Earlier dropouts among HAS participants may suggest that those with elevated symptoms found it more difficult to engage in the program. Nevertheless, the two classes had similar levels of initial engagement (based on activity completion), suggesting that other factors, such as perceived treatment efficacy, may have influenced adherence.
The implications of this research's findings are not limited to the BRAVE program and provide useful insights for other web-based interventions. For example, when there is a broad range of users who engage with an internet intervention, with diverse profiles, particularly related to symptom severity, the results of this study indicate that care should be taken to consider the needs of these subgroups. Particular consideration should be given to whether the service model is appropriate for them, whether the service needs to be tailored to profiles (eg, with additional support), or whether the service needs to be targeted to those who will benefit the most. However, it is important to note that there are drawbacks to targeting more narrowly - there is still a clear demand for the BRAVE program even among those in the high severity class, which suggests that many young people with anxiety may not be receiving the care they need from other sources (whether web-based or in-person).

\section{Strengths and Limitations}

There are a number of clear strengths of this study. It had a large sample size, included both children and adolescents, used naturalistic data from a clinical service without stringent eligibility criteria, and collected symptom-level data throughout the completion of the program. However, alongside these strengths, this study has a number of limitations. High attrition was observed, and although models were robust to missing data, dropout may be associated with treatment response. All outcome measures were self-reported; thus, there was no clinical verification of the symptom level. Furthermore, as the data were collected in the context of clinical service delivery, there was no control group. This makes it unclear whether factors external to the program may have influenced the trajectories and whether the reductions in anxiety were attributed to the treatment or were the result of spontaneous recovery over time. Finally, limited psychosocial factors were measured; thus, other factors, such as ruminative style, personality, social support, and previous exposure to treatment, may have influenced the observed trajectories.

\section{Future Research Directions}

Recent worldwide events have resulted in a significant shift in mental health care service needs, as numerous lockdowns during the COVID-19 pandemic severely impacted face-to-face mental health service access and provision. This subsequently led to the conversion of many services to telehealth, videoconferencing, and other digital modalities to provide continuity of care. With an increased access to digital mental health services, and likely increased experiences of distress at the community level, it will be important to examine whether the use of the BRAVE Program changes throughout and after COVID-19. Future research should examine whether users presenting to the web-based program during and after the pandemic present with different profiles and symptom presentations and whether their experience with the web-based program leads to similar outcomes as standard BRAVE program users.

\section{Conclusions}

Young people completing the BRAVE Self-Help program tend to fall into two trajectory classes that can be reliably identified 
in terms of high versus moderate baseline levels of anxiety and subsequent reductions in symptoms. Both classes showed significant reductions in anxiety, with the greatest level of change being achieved within the first six sessions for both classes. Those in the MAS class tended to show reductions in anxiety symptoms to levels below the elevated range, whereas those in the high symptom group tended to remain in the elevated range despite improvements. Thus, those in the high severity group who do not respond well to iCBT on a self-help basis may benefit from a stepped-care approach or additional support.

\section{Acknowledgments}

The authors wish to acknowledge the initial funders of the BRAVE Self-Help program, beyondblue; the many participants who participated in this research; the research assistants working on the project; and the members of the advisory panel (Professor David Kavanagh, Professor Jane Burns, Professor Britt Klein, beyondblue, and blueVoices youth representatives) who provided guidance on the overall implementation of the initial BRAVE Self-Help program. Authors SM, SHS, CD, AR, and ALC acknowledge funding provided by the Medical Research Future Fund through the Department of Health (APP1179490), and ALC acknowledges funding provided by the National Health and Medical Research Council fellowship (1173146).

\section{Conflicts of Interest}

SM, SHS, and CD acknowledge that although intellectual property for BRAVE-ONLINE is owned by UniQuest, they may potentially benefit from future royalties related to the program.

\section{Multimedia Appendix 1}

Breakdown of referral sources to the BRAVE self-help program $(\mathrm{N}=10,366)$.

[DOCX File, 23 KB-Multimedia Appendix 1]

\section{References}

1. Lawrence D, Johnson S, Hafekost J, Boterhoven de Haan K, Sawyer M, Ainley J, et al. The Mental Health of Children and Adolescents. Report on the second Australian Child and Adolescent Survey of Mental Health and Wellbeing. 2015. URL: https://www.health.gov.au/sites/default/files/documents/2020/11/the-mental-health-of-children-and-adolescents $0 . p d f$ [accessed 2021-06-04]

2. Australian Government Response to Contributing Lives, Thriving Communities - Review of Mental Health Programmes and Services. Australian Government - Department of Health. 2015. URL: https://www.health.gov.au/sites/default/files/ response-review-of-mental-health-programmes-and-services.pdf [accessed 2021-06-04]

3. Donovan CL, March S. Online CBT for preschool anxiety disorders: a randomised control trial. Behav Res Ther 2014 Jul;58:24-35. [doi: 10.1016/j.brat.2014.05.001] [Medline: 24927471]

4. March S, Spence SH, Donovan CL. The efficacy of an internet-based cognitive-behavioral therapy intervention for child anxiety disorders. J Pediatr Psychol 2009 Jun 15;34(5):474-487. [doi: 10.1093/jpepsy/jsn099] [Medline: 18794187]

5. Reyes-Portillo JA, Mufson L, Greenhill LL, Gould MS, Fisher PW, Tarlow N, et al. Web-based interventions for youth internalizing problems: a systematic review. J Am Acad Child Adolesc Psychiatry 2014 Dec;53(12):1254-70.e5. [doi: 10.1016/j.jaac.2014.09.005] [Medline: 25457924]

6. Spence SH, Donovan CL, March S, Gamble A, Anderson RE, Prosser S, et al. A randomized controlled trial of online versus clinic-based CBT for adolescent anxiety. J Consult Clin Psychol 2011 Oct;79(5):629-642. [doi: 10.1037/a0024512] [Medline: 21744945]

7. Spence SH, Holmes JM, March S, Lipp OV. The feasibility and outcome of clinic plus internet delivery of cognitive-behavior therapy for childhood anxiety. J Consult Clin Psychol 2006;74(3):614-621. [doi: 10.1037/0022-006x.74.3.614] [Medline: 16822117]

8. Warwick H, Reardon T, Cooper P, Murayama K, Reynolds S, Wilson C, et al. Complete recovery from anxiety disorders following cognitive behavior therapy in children and adolescents: a meta-analysis. Clin Psychol Rev 2017 Mar;52:77-91. [doi: 10.1016/j.cpr.2016.12.002] [Medline: 28040627]

9. March S, Spence SH, Donovan CL, Kenardy JA. Large-scale dissemination of internet-based cognitive behavioral therapy for youth anxiety: feasibility and acceptability study. J Med Internet Res 2018 Jul 4;20(7):e234 [FREE Full text] [doi: 10.2196/jmir.9211] [Medline: 29973338]

10. Christensen H, Griffiths KM, Farrer L. Adherence in internet interventions for anxiety and depression. J Med Internet Res 2009 Apr 24;11(2):e13 [FREE Full text] [doi: 10.2196/jmir.1194] [Medline: 19403466]

11. Vigerland S, Ljótsson B, Thulin U, Öst LG, Andersson G, Serlachius E. Internet-delivered cognitive behavioural therapy for children with anxiety disorders: a randomised controlled trial. Behav Res Ther 2016 Jan;76:47-56 [FREE Full text] [doi: 10.1016/j.brat.2015.11.006] [Medline: 26649465] 
12. Jolstedt M, Ljótsson B, Fredlander S, Tedgård T, Hallberg A, Ekeljung A, et al. Implementation of internet-delivered CBT for children with anxiety disorders in a rural area: a feasibility trial. Internet Interv 2018 Jun;12:121-129 [FREE Full text] [doi: 10.1016/j.invent.2017.11.003] [Medline: 30135776]

13. Stasiak K, Merry SN, Frampton C, Moor S. Delivering solid treatments on shaky ground: feasibility study of an online therapy for child anxiety in the aftermath of a natural disaster. Psychother Res 2018 Jul 26;28(4):643-653. [doi: 10.1080/10503307.2016.1244617] [Medline: 27781568]

14. Titov N, Dear BF, Nielssen O, Wootton B, Kayrouz R, Karin E, et al. User characteristics and outcomes from a national digital mental health service: an observational study of registrants of the Australian MindSpot clinic. Lancet Digit Health 2020 Nov;2(11):e582-e593. [doi: 10.1016/s2589-7500(20)30224-7] [Medline: 33103097]

15. Titov N, Andrews G, Schwencke G, Robinson E, Peters L, Spence J. Randomized controlled trial of Internet cognitive behavioural treatment for social phobia with and without motivational enhancement strategies. Aust N Z J Psychiatry 2010 Oct;44(10):938-945. [doi: 10.3109/00048674.2010.493859] [Medline: 20932208]

16. Ritterband LM, Thorndike FP, Gonder-Frederick LA, Magee JC, Bailey ET, Saylor DK, et al. Efficacy of an internet-based behavioral intervention for adults with insomnia. Arch Gen Psychiatry 2009 Jul 1;66(7):692-698 [FREE Full text] [doi: 10.1001/archgenpsychiatry.2009.66] [Medline: 19581560]

17. Batterham PJ, Christensen H, Mackinnon AJ, Gosling JA, Thorndike FP, Ritterband LM, et al. Trajectories of change and long-term outcomes in a randomised controlled trial of internet-based insomnia treatment to prevent depression. BJPsych Open 2017 Sep 2;3(5):228-235 [FREE Full text] [doi: 10.1192/bjpo.bp.117.005231] [Medline: 28959453]

18. Christensen H, Griffiths KM, MacKinnon AJ, BritteLiffee K. Online randomized controlled trial of brief and full cognitive behaviour therapy for depression. Psychol Med 2006 Aug 29;36(12):1737-1746. [doi: 10.1017/s0033291706008695]

19. Chu BC, Skriner LC, Zandberg LJ. Shape of change in cognitive behavioral therapy for youth anxiety: symptom trajectory and predictors of change. J Consult Clin Psychol 2013 Aug;81(4):573-587. [doi: 10.1037/a0033390] [Medline: 23796317]

20. Queen AH, Barlow DH, Ehrenreich-May J. The trajectories of adolescent anxiety and depressive symptoms over the course of a transdiagnostic treatment. J Anxiety Disord 2014 Aug;28(6):511-521. [doi: 10.1016/j.janxdis.2014.05.007] [Medline: 24960439]

21. Batterham PJ, van Spijker BBA, Mackinnon AJ, Calear AL, Wong Q, Christensen H. Consistency of trajectories of suicidal ideation and depression symptoms: evidence from a randomized controlled trial. Depress Anxiety 2019 Apr 22;36(4):321-329. [doi: 10.1002/da.22863] [Medline: 30466148]

22. Crocetti E, Klimstra T, Keijsers L, Hale WW, Meeus W. Anxiety trajectories and identity development in adolescence: a five-wave longitudinal study. J Youth Adolesc 2009 Jul 13;38(6):839-849. [doi: 10.1007/s10964-008-9302-y] [Medline: 19636785]

23. Olino TM, Klein DN, Lewinsohn PM, Rohde P, Seeley JR. Latent trajectory classes of depressive and anxiety disorders from adolescence to adulthood: descriptions of classes and associations with risk factors. Compr Psychiatry 2010 May;51(3):224-235 [FREE Full text] [doi: 10.1016/j.comppsych.2009.07.002] [Medline: 20399331]

24. Spence SH, Donovan CL, March S, Gamble A, Anderson R, Prosser S, et al. Online CBT in the treatment of child and adolescent anxiety disorders: issues in the development of brave-online and two case illustrations. Behav Cogn Psychother 2008 Jul 1;36(4):411-430. [doi: 10.1017/s135246580800444x]

25. Spence S, Sawyer M, Sheffield J, Patton G, Bond L, Graetz B, et al. Does the absence of a supportive family environment influence the outcome of a universal intervention for the prevention of depression? Int J Environ Res Public Health 2014 May 13;11(5):5113-5132 [FREE Full text] [doi: 10.3390/ijerph110505113] [Medline: 24828082]

26. Australian Standard Geographical Classification (ASGC). Australian Bureau of Statistics. 2011. URL: https://www. abs.gov.au/websitedbs/d3310114.nsf/home/australian+standard+geographical+classification+(asgc) [accessed 2021-06-04]

27. Muthen B, Muthen LK. Integrating Person-Centered and Variable-Centered Analyses: Growth Mixture Modeling With Latent Trajectory Classes. Alcoholism Clin Exp Res 2000 Jun;24(6):882-891. [doi: 10.1111/j.1530-0277.2000.tb02070.x]

28. Nylund KL, Asparouhov T, Muthén BO. Deciding on the number of classes in latent class analysis and growth mixture modeling: a Monte Carlo simulation study. Struct Equat Model: Multidiscip J 2007 Dec 5;14(4):535-569. [doi: 10.1080/10705510701575396]

29. March S, Donovan CL, Baldwin S, Ford M, Spence SH. Using stepped-care approaches within internet-based interventions for youth anxiety: three case studies. Internet Interv 2019 Dec;18:100281 [FREE Full text] [doi: 10.1016/j.invent.2019.100281] [Medline: $\underline{31890629]}$

30. Merikangas KR, Nakamura EF, Kessler RC. Epidemiology of mental disorders in children and adolescents. Dialogues Clin Neurosci 2009;11(1):7-20 [FREE Full text] [Medline: 19432384]

\section{Abbreviations}

BLRT: Bootstrapped Likelihood Ratio Test

CAS-8: Children's Anxiety Scale 8-item

CBT: cognitive behavioral therapy

GMM: growth mixture model 
HAS: high anxiety severity

iCBT: internet-based cognitive behavior therapy

MAS: moderate anxiety severity

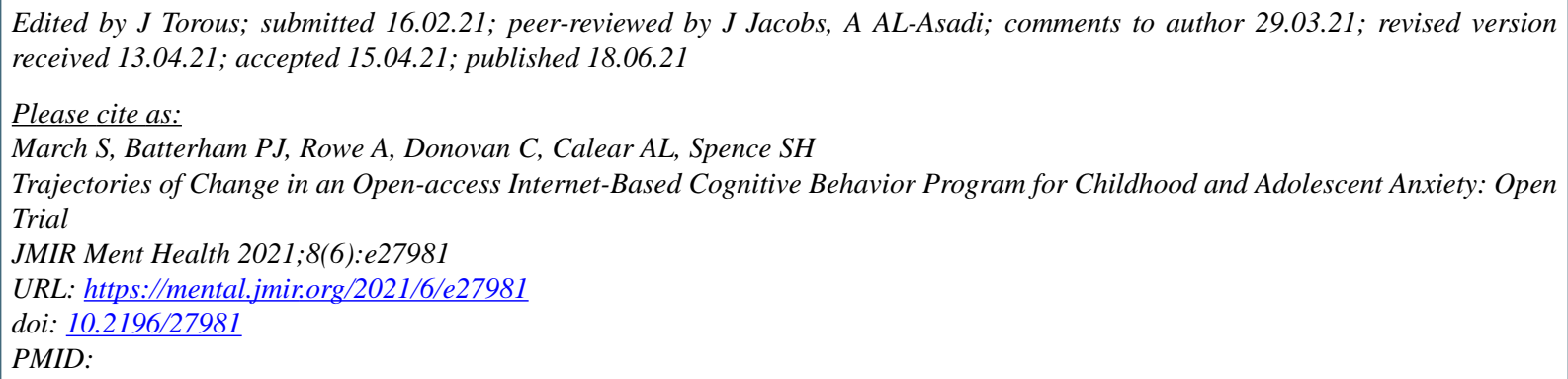

(C) Sonja March, Philip J Batterham, Arlen Rowe, Caroline Donovan, Alison L Calear, Susan H Spence. Originally published in JMIR Mental Health (https://mental.jmir.org), 18.06.2021. This is an open-access article distributed under the terms of the Creative Commons Attribution License (https://creativecommons.org/licenses/by/4.0/), which permits unrestricted use, distribution, and reproduction in any medium, provided the original work, first published in JMIR Mental Health, is properly cited. The complete bibliographic information, a link to the original publication on https://mental.jmir.org/, as well as this copyright and license information must be included. 